\title{
Worin besteht das Germanistische der Germanistik (und worin nicht)?
}

Der Call for Papers zu diesem Themenheft stellt in vielfältigen Facetten die Fragen, ob es noch eine Einheit des Fachs »Germanistik« gibt, geben sollte, geben kann und welche Konsequenzen und Aussichten sich aus einer positiven wie negativen Antwort ergeben.

Bei näherem Hinsehen aber stellt sich heraus, dass diese Fragen eigentlich gar nicht so artikuliert sind, dass sie sich spezifisch auf die Germanistik beziehen. Vielmehr schienen sie alle Philologien, den Zusammenhang zwischen Sprach- und Literaturwissenschaften ganz allgemein zu betreffen. Der CfP benennt nämlich keine Problemstellung oder Situation, die spezifisch germanistisch wäre. Das überrascht zumindest zunächst ein wenig.

Gibt es noch (sinnvoller-, notwendigerweise) eine Sprachraumphilologie? (»Nationalphilologie« will ich wegen des chauvinistischen Beiklangs nicht sagen.) Sollte es, muss es sie geben? Die Diskussionen, die man hier führen müsste, würden in ganz andere Richtungen führen, die vom CfP nicht intendiert sind. Welche Rolle spielt das Deutsche in der Welt, welche Rolle spielt es als Einzelsprache? Welchen Stellenwert hat Literatur in der Kultur? Welchen haben Literatur und Medien bei der Erkenntnis sprachlicher Tatsachen? Was muss man über D, A, CH (auch I, B, LUX, FL, F) und ihre Geschichte wissen, um deutsche Kultur, Kommunikation und Sprache zu verstehen? Und schließlich: Was wird aus der Germanistik unter dem zunehmenden Imperativ der englischen Publikation? Kann es noch eine bedeutende Germanistik geben, wenn Deutsch so langsam selbst in der Germanistik nicht mehr die primäre, zumindest nicht mehr die Wissenschaftssprache für reputierte Veröffentlichungen ist und wenn kaum noch autochthone wissenschaftliche Begriffsbildung in der Objektsprache als Metasprache des Fach geschieht? Dies sind Fragen, die vielleicht gewissen kulturwissenschaftlichen, aber übrigens genauso auch generativistischen Ohren chauvinistisch, provinzialistisch oder anderweitig unzeitgemäß klingen. Für die Frage nach der Identität und Zukunft einer »Germanistik «, ob nun als integriertes Fach oder als unabhängig voneinander institutionalisierte, forschende und lehrende germanistische Sprach- und Literaturwissenschaft, rühren die möglichen Antworten aber an die Substanz des Fachs. Welchen Status das Deutsche, sowohl als Objektsprache, die den Gegenstand unserer Untersuchungen ausmacht, als auch als wissenschaftliche Metasprache, in Zukunft bei der Befassung mit deutscher Sprache und Literatur einnehmen wird, wird darü- 
ber entscheiden, ob wir zukünftig noch von der »Germanistik « reden werden, oder ob sie zumindest zu weiten Teilen von allgemeineren sprach-, kommunikations-, kultur-, literatur- und medienwissenschaftlichen Studiengängen, Professuren und Instituten absorbiert wird.

Geht es nun aber jenseits dieser Fragen, die vor allem wissenschaftspolitische und teils darüber hinaus reichende gesellschaftspolitische Dimensionen betreffen, darum, eine »Vision « der theoretischen und methodologischen Ausrichtung des Fachs vorzuschlagen, wie dies der CfP anregt, dann schient mir Vorsicht angeraten. Ich möchte mich vor allem auf meine Erfahrungen aus der Arbeit im Begutachtungsprozess der DFG im Fachkollegium »Sprachwissenschaften «, in dem ich in den letzten sechs Jahren tätig sein durfte, beziehen. In diesem kompetitiven Kontext spielen die Traditionen der Einzelphilologien eine eher geringe Rolle. Bezüge zur Literaturwissenschaft und Vorhaben, die Sprach- und Literaturwissenschaftliches verbinden, gibt es so gut wie keine. Die Arbeit im DFG-Fachkolleg konfrontiert mit der Breite der Arten und Weisen, wie man das Fach (sei es nun »Sprachwissenschaft« oder »Germanistik «) verstehen kann, wie man gegenstandsbezogene, methodologische und theoretische Prioritäten setzen kann. Der Gegenstände und Ansätze sind so immens viele, viele von ihnen zeitigen hervorragende Resultate und sind jeweils ausgezeichnet legitimierbar - natürlich manche (m.E.) auch weniger. Die Vielfalt und Breite von unterschiedlichsten, von naturwissenschaftlichbiologischen, über experimentell-kognitive, korpusbasiert-quantitative, kulturwissenschaftlich-interpretative bis hin zu deskriptiv-dokumentierenden Vorhaben, die in je eigener Weise gewachsenen, fachlich ausgebildeten Standards folgen, legen nahe, vorsichtig zu sein, eine Position dazu zu beziehen, wohin sich »das Fach « entwickeln kann oder soll. Dies ist beileibe kein Plädoyer für ein anything goes und auch keine Resignation vor Überkomplexität. Die Komplexität des Gegenstands, die eine ausdifferenzierte Wissenschaft heute repräsentieren und in ihren Fragestellungen und Methoden praktizieren können muss, erzwingt eine notwendige und legitime Vielfalt der Zugänge. In anderen professionell ausdifferenzierten Fächern verhält es sich nicht anders. Alles andere wäre ein Armutszeugnis für das Fach. Dafür zu kämpfen, dies zu vertreten, lohnt sich. Und die Tatsache, dass die Sprachwissenschaften im Bereich der Forschungsförderung recht gut dastehen, hängt sicher auch mit der Tatsache zusammen, dass sie es schaffen, faktisch koexistierend und manchmal auch inhaltlich synergetisch verknüpfend verschiedenste Perspektiven auf ihre Gegenstände auf hohem wissenschaftlichem Niveau beständig weiterzuentwickeln. Sicher ist es berechtigt und politisch manchmal unumgänglich, durchaus auch mal polemisch für ein bestimmtes Anliegen zu streiten. Doch wollte man einer Wissenschaftslandschaft als ganzer eine Ausrichtung anempfehlen, wäre die Gefahr allzu groß, dass dies eher von Unwissen, ideologischem Eifer oder orthodoxer Beschränktheit zeugte, als dass damit tatsächlicher Erkenntnisfortschritt zu gewinnen wäre. 